\title{
CLINICAL PRACTICE Clinical Vignette Smear Campaign: Misattribution of Pancytopenia to a Tick-Borne Illness
}

\author{
Jessica Lee, MD ${ }^{1,2}$, Soraya Azzawi ${ }^{7}$, Michael J. Peluso, MD ${ }^{1,2}$, Aaron Richterman, MD ${ }^{1,2}$, \\ Haiyan Ramirez Batlle, $M D^{1,2}$, and Maria A. Yialamas, $M D^{1,2,3}$ \\ ${ }^{1}$ Harvard Medical School, Boston, MA, UA; ${ }^{2}$ Department of Medicine, Brigham and Women's Hospital, Boston, MA, USA; ${ }^{3}$ nternal Medicine \\ Residency Program, Boston, MA, USA.
}

We report the case of a 51-year-old woman presenting with a targetoid rash and pancytopenia after a tick bite. Initial evaluation was notable for severe neutropenia on the complete blood cell count differential, a positive Lyme IgM antibody, and a peripheral blood smear demonstrating atypical lymphocytes. While her pancytopenia was initially attributed to tick-borne illness, peripheral flow cytometry showed 7\% myeloblasts, and a bone marrow biopsy confirmed $60 \%$ blasts. The patient was ultimately diagnosed with acute myelogenous leukemia, in addition to early, localized Lyme disease. This case highlights the differential diagnosis for pancytopenia, cytopenia patterns for different tick-borne illnesses, the risk of premature closure in internal medicine, and management of Lyme disease in hosts with altered immunity.

KEY WORDS: pancytopenia; tick-borne illness; Lyme disease; acute myelogenous leukemia.

J Gen Intern Med 33(4):570-2

DOI: $10.1007 /$ s11606-017-4241-5

(C) Society of General Internal Medicine 2017

\section{INTRODUCTION}

Cytopenias can occur in patients with tick-borne illnesses, and development of such illnesses following a tick bite is common in areas of the United States endemic for these diseases. ${ }^{1,2}$ However, the time course and severity of cytopenia differ in the various tick-borne infections, ${ }^{3-7}$ and it is important to consider the differential diagnosis for pancytopenia and to avoid premature closure, ${ }^{8,9}$ even in cases of known tick exposure. Here, we present the case of a woman who presented with a witnessed tick exposure and was found to have pancytopenia, which was initially attributed to a tick-borne illness. Further evaluation revealed an undiagnosed underlying malignancy as the cause of her pancytopenia, which in turn led to questions regarding the appropriate management of her concomitant tick-borne illness.

Received June 30, 2017

Revised October 4, 2017

Accepted November 17, 2017

Published online December 22, 2017

\section{Case}

A 51-year-old woman with a history of fibromyalgia presented with a rash following a tick bite that she sustained while visiting Martha's Vineyard in the summer. The day after finding a tick attached to her right arm, she noticed a tender, erythematous, targetoid rash on her left calf. She presented to a local hospital, where laboratory investigation revealed a white blood cell $(\mathrm{WBC})$ count of $0.7 \times 10^{3}$ cells $/ \mu \mathrm{L}$, hemoglobin of $9.6 \mathrm{~g} / \mathrm{L}$, and platelet count of $85 \times 10^{6}$ cells $/ \mu \mathrm{L}$; the white blood cell differential showed $3 \%$ neutrophils, $89 \%$ lymphocytes, $7 \%$ monocytes, and $1 \%$ eosinophils. The tick had been discarded and therefore was unavailable for identification. Doxycycline was initiated. The following day, her pancytopenia persisted and her leukopenia worsened (WBC $0.5 \times 10^{3}$ cells $/ \mu \mathrm{L}$, absolute neutrophil count 10 cells/ $\mu \mathrm{L}$ ). Her pancytopenia was attributed to a tick-borne infection and she was transferred to a tertiary medical center for further evaluation and management

On arrival, her temperature was 100 degrees Fahrenheit, heart rate was 105 beats per minute, blood pressure was $125 / 75 \mathrm{mmHg}$, with a normal respiratory rate and oxygen saturation. She appeared comfortable and well-nourished, and she had an erythematous, targetoid rash on her left calf. Cardiovascular, abdominal, and neurologic exams were normal. She had no effusions, erythema, or pain associated with any of her joints, and there were no petechiae present. A tick-borne disease panel returned with positive Lyme IgM, and studies were negative for Babesia, Ehrlichia/Anaplasma, and Rickettsia. A manual peripheral blood smear demonstrated poikilocytosis, occasional teardrop cells, thrombocytopenia, atypical lymphocytes, and no neutrophils (Fig. 1). Studies for HIV, parvovirus B19, Epstein-Barr virus (EBV), Cytomegalovirus (CMV), and blood and urine cultures were negative.

Upon further questioning, the patient endorsed months of malaise, easy bruising, and occasional night sweats. Based on this history and the peripheral blood smear, peripheral flow cytometry was performed, and revealed $7 \%$ myeloblasts. A follow-up bone marrow biopsy demonstrated $60 \%$ blasts. Ultimately, the patient was diagnosed with acute myelogenous leukemia (AML), in addition to early, localized Lyme disease. She was treated with 


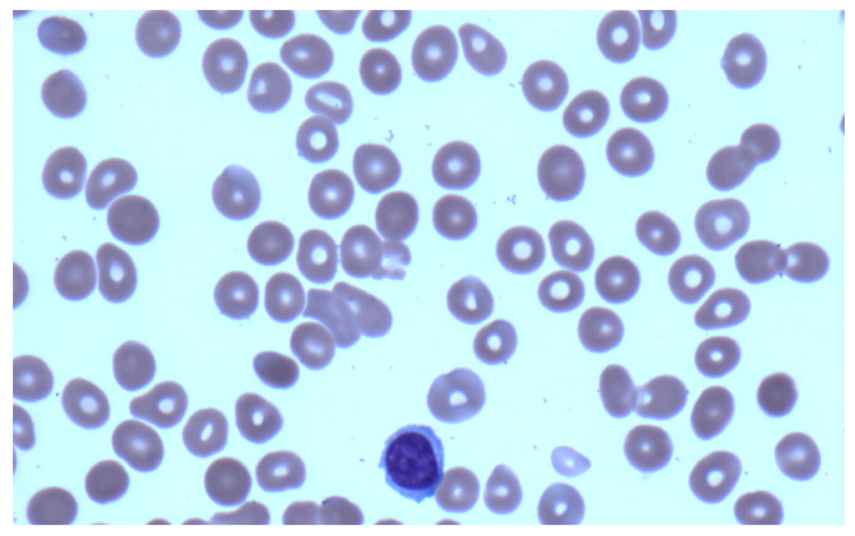

Figure 1 High-powered $(\times 100)$ view of the patient's peripheral blood smear at the time of presentation as a transfer from the outside hospital. Occasional teardrop cells, decreased platelets, and an atypical lymphocyte can be seen in this field. Acknowledgement: Maureen Achabe and Elisa Aquilanti.

a 14-day course of doxycycline while receiving induction chemotherapy with a regimen of cytarabine and daunorubicin. She tolerated her antibiotic treatment well and did not develop advanced Lyme disease; she eventually underwent a bone marrow transplant for management of AML.

\section{DISCUSSION}

While pancytopenia is known to occur in tick-borne illness, the degree of pancytopenia, in particular neutropenia, in this case would be atypical for this etiology and called for a broader workup. The differential diagnosis for acquired pancytopenia can be divided into systemic and bone marrow causes (Fig. 2). ${ }^{10,11}$ Systemic causes include infection (in particular parvovirus, HIV, hepatitis, CMV, and EBV), sepsis, medication effect, nutritional deficiencies, alcoholism, and autoimmune conditions such as systemic lupus erythematosus. Bone marrow etiologies can be divided into causes associated with hypocellular or cellular marrow. Causes associated with hypocellular marrow include aplastic anemia and hypoplastic myelodysplastic syndrome, while those associated with cellular marrow comprise hematopoietic disorders (myelodysplastic syndrome, leukemia or lymphoma, or paroxysmal nocturnal hemoglobinuria) and metastatic or granulomatous disease that leads to marrow replacement. ${ }^{10}$ Cytopenias from unrelated causes can also combine to present as pancytopenia. The peripheral blood smear is an essential component of the diagnostic workup for pancytopenia, even in cases in which the etiology is thought to be understood. In this case, pancytopenia was initially thought to be due to tick-borne illness, but the blood smear

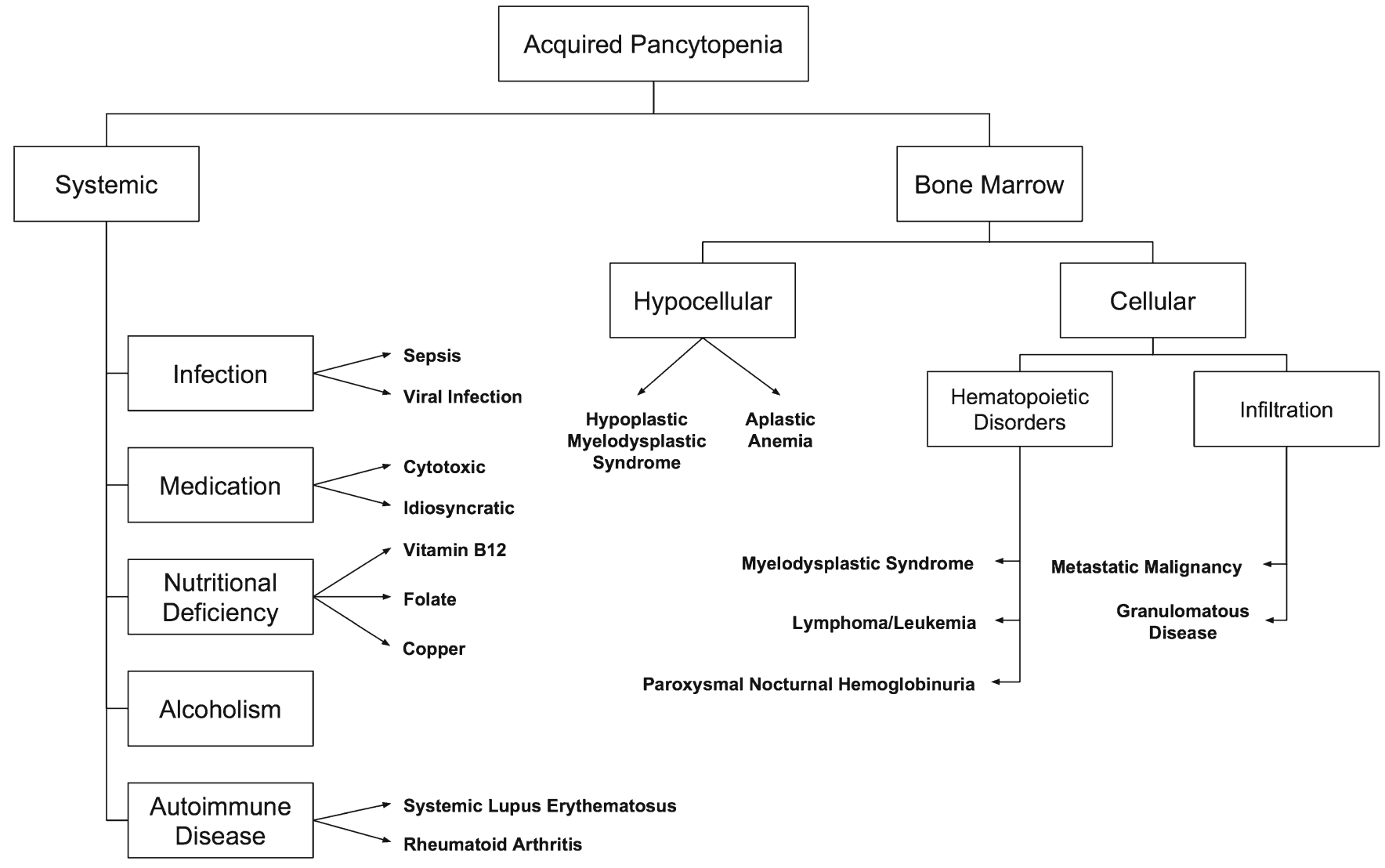

Figure 2 The differential diagnosis for acquired pancytopenia. 
suggested the possibility of malignancy and prompted further testing, which revealed AML.

Tick-borne disease can be associated with cytopenias, particularly in Babesia and Anaplasma infections. In a review of 51 patients with babesiosis, anemia and thrombocytopenia were frequent, and $35 \%$ of patients experienced neutropenia at some point during their clinical course. ${ }^{4}$ Among 44 patients with anaplasmosis, 55\% were leukopenic, $36 \%$ anemic, and $61 \%$ thrombocytopenic. ${ }^{5}$ In the same study, among the 62 patients with Lyme disease alone, only $7 \%$ were leukopenic, $8 \%$ anemic, and $10 \%$ thrombocytopenic. ${ }^{5}$ Similar results have been found in other cohorts. ${ }^{3,7}$ This suggests that cytopenias associated with Lyme disease alone are relatively rare, and more commonly are present when Lyme disease co-occurs with Babesia and/or Anaplasma infection, which were not present in this case. ${ }^{7}$

There is scant literature describing the effects of altered immunity on the course of Lyme disease. In a prospective study of patients with erythema migrans, the rash classically associated with Lyme disease, the 53 patients with underlying hematologic malignancy were more likely to have signs of disseminated Lyme disease and more frequently required antibiotic retreatment than immunocompetent controls. ${ }^{12}$ A retrospective study of patients with erythema migrans found that prevalence of IgG and IgM antibodies was similar between patients who were immunocompromised (33 patients) and those who were immunocompetent (75 patients), and there were no differences in the outcomes of early Lyme disease. ${ }^{13}$ There was, however, a difference in the location of erythema migrans. Immunocompetent patients were more likely to experience the rash on their lower extremities $(69 \%)$ than were immunocompromised patients, who had a broader distribution, including the trunk $(37 \%)$ in addition to lower extremities $(33 \%)$. There is no standardized antibiotic regimen for treatment of Lyme in an immunocompromised patient. In this case, the medical team elected for a typical treatment course with careful monitoring after consultation with the infectious disease consultant team.

\section{CONCLUSIONS}

Pancytopenia is a rare manifestation of Lyme disease and should prompt evaluation for alternate processes, including co-infection with Anaplasma and/or Babesia, or other causes such as hematologic malignancy. Immunocompromised patients may present with different and disseminated manifestations of Lyme disease, and may be more likely to require retreatment, but there is currently no evidence to support a different therapeutic approach.

\section{Acknowledgments:}

Contributors: The authors acknowledge Maureen Achabe and Elisa Aquilanti for providing the photograph of the peripheral blood smear.

Corresponding Author: Maria A. Yialamas, MD; Department of Medicine, Brigham and Women's Hospital, Boston, MA, USA (e-mail: myialamas@bwh.harvard.edu).

\section{Compliance with Ethical Standards:}

Prior Presentations: This work was presented as an oral presentation at the Society of General Internal Medicine New England Regional Meeting, Boston, MA, March 2017, and as a poster presentation at the Society of General Internal Medicine National Meeting, Washington, DC, April 2017.

Conflict of Interest: All authors declare that they have no conflict of interest.

\section{REFERENCES}

1. U.S. Department of Health and Human Services Centers for Disease Control and Prevention. Tickborne diseases of the United States: A Reference Manual for Health Care Providers. 4th edition. 2017. Available at: https://www.cdc.gov/lyme/resources/tickbornediseases.pdf. Accessed 27 June 2017.

2. Hook SA, Nelson CA, Mead PS. U.S. public's experience with ticks and tick-borne illnesses: results from national HealthStyles surveys. Ticks Tick Borne Dis. 2015; 6(4):483-8.

3. Krause OJ, McKay K, Thompson CA, et al. Disease-specific diagnosis of coinfecting tickborne zoonoses: babesiosis, human granulocytic ehrlichiosis, and Lyme disease. Clin Infect Dis. 2002;34(9):1184-91.

4. Wormser GP, Villafuerte $\mathbf{P}$, Nolan SM, et al. Neutropenia in congenital and adult babesiosis. Am J Clin Pathol. 2015;144(1):94-6.

5. Wormser GP, Aguero-Rosenfeld ME, Cox ME, et al. Differences and similarities between culture-confirmed human granulocytic anaplasmosis and early Lyme disease. J Clin Microbiol. 2013;51(3):954-8.

6. Dumler JS, Madigan JE, Pusterla N, Bakken JS. Ehrlichioses in humans: epidemiology, clinical presentation, diagnosis, and treatment. Clin Infect Dis. 2007;45(Suppl 1):S45-51.

7. Nadelman RB, Nowakowski J, Forseter G, et al. The clinical spectrum of early Lyme borreliosis in patients with culture-confirmed erythema migrans. Am J Med. 1996;100(5):502.

8. Borrell-Carrio F, Epstein RM. Preventing errors in clinical practice: a call for self-awareness. Ann Fam Med. 2004;2:310-6.

9. McSherry D. Avoiding premature closure in sequential diagnosis. Artif Intell Med. 1997;10(3):269-83.

10. Weinzierl EP, Arber DA. The differential diagnosis and bone marrow evaluation of new-onset pancytopenia. Am J Clin Pathol. 2013;139(1):9-29.

11. Jain A, Naniwadekar M. An etiological reappraisal of pancytopenialargest series reported to date from a single tertiary care teaching hospital. BMC Hematol. 2013;13(1):10.

12. Maraspin V, Ružić-Sabljić E, Lusa L, Strle F. Course and outcome of Early Lyme borreliosis in patients with hematological malignancies. Clin Infect Dis. 2015;61(3):427.

13. Furst B, Glatz M, Kerl H, Mullegger RR. The impact of immunosuppression on erythema migrans. A retrospective study of clinical presentation, response to treatment and production of Borrelia antibodies in 33 patients. Clin Exp Dermatol. 2006;31(5)751. 